\title{
Electron Microscopy of Nerve Fibers VIII.
}

\author{
Again on the Radial Component in the Myelin Sheath
}

\author{
by \\ Ryohei Honjin* and George W. Changus \\ The Section of Experimental Histology, Mercy Hospital, \\ Laboratory, Chicago, Illinois, U.S.A.
}

Recently the existence of a radially oriented dense structure running through the myelin sheath has been shown by $\mathrm{Honjin}$ (1959), R obertson (1960), Peters $(1961,1962)$ and $\mathrm{Honjin}$, $\mathrm{Kos}$ a ka, T a k a $\mathrm{no}$ and $\mathrm{H}$ ir a mats u (1963) with electron microscopy. The radial component of the myelin sheath is of undetermined significance. Moreover the descriptions about it have been limited to a few kinds of animals. The purpose of the present article is to describe the occurrence and characteristics of the radial component in myelin sheath from the optic nerve of a species of frog, Rana pipiens, to complement the previous knowledge about it in the other animals.

\section{Materials and Methods}

The materials for the present study were taken from the optic nerves of adult frogs (Rana pipiens) of both sexes. Small pieces of nerve were quickly removed from the animals and fixed 2 to 4 hours in a vial of fixative immersed in ice water. Both veronal acetate-buffered $1 \% \mathrm{OsO}_{4}\left(\mathrm{Pal}\right.$ a de, 1952) and $0.6 \% \mathrm{KMnO}_{4}(\mathrm{~L} \mathrm{u} \mathrm{ft}$, 1956) at $\mathrm{pH} 7.42$ were used as fixatives. Dehydration was in graded concentrations of ethanol from 30 to $100 \%$. The materials were then embedded in either $7: 1$ mixture of butyl and methyl methacrylates or epoxy resin (Araldite) according to the procedure of $\mathrm{Gl}$ a u e $\mathrm{r}$ and G l a u e r t (1958), and polymerized in gelatin capsules at $60^{\circ} \mathrm{C}$. overnight. Thin sections were cut on a LKB ultratome

* Present adress: Department of Anatomy, School of Medicine, University of Kanazawa, Kanazawa, Japan. Dr. Honjin was an exchange visiting professor in Mercy Chicago's First Hospital. 
using glass knives, and were mounted on collodion-covered copper grids. Some of the sections were stained with either potassium permanganate method by L a w n (1960) or plumbite ion method by $\mathrm{K}$ a r now s k y (1961). Observations were made by a HU-11-A type electron microscope with $20 \mu$ objective aperture at $75 \mathrm{kV}$. Electron micrographs were made at original magnifications of 15,000 to 50,000 times and subsequently enlarged photographically. For light microscopy, thick sections of plastic embedded materials were mounted on glass slides and examined without removing the plastic.

\section{Observations}

The radial components are found in some of the myelinated nerve fibers of this species of frogs. However it must be emphasized that the radial components are not always present in every myelin sheath, though they occur quite regullary in some of the myelin sheaths which are sporadically distributed throughout the optic nerve. Many myelin sheaths in thin sections do not show any radial components. The materials embedded in methacrylate are convenient for finding out the radial components, particularly in case of the non-stained sections, on account of its feature of relatively high contrast of images in electron micrographs. However, generally speaking, methacrylate embedding did not produce good results, because it brought about the destruction of the lamellar structure of myelin sheath, owing to shrinking effect at the time of polymerization. In the present study a combination of both embedding by epoxy resin and staining by plumbic ion produced good results for observing the radial components, because of the excellent preservation of the myelin lamellae and of the suitable contrast of the images.

The radial components which are cut perpendicularly to the myelin lamellar layers, appear as a series of small electron-dense thickenings. The thickenings situated in the interperiod lines of the lamellated myelin sheath and stand in several rows arranged radially across the thickness of the myelin sheath (Figs. 1 to 7 ). When the sheaths showing the radial component are sectioned in transverse or parallel with axis of the nerve fiber, the pattern of the rows of radial components can be observed arranged radially across the thickness of the sheath at right angles to the local region of the myelin lamellae (Figs. 1, 3, 4), while when the myelin sheaths are cut obliquely to the axis of the nerve fiber, the rows of radial 
components show somewhat oblique locus passing through the myelin layers (Figs. 2, 5, 6,7). The dense period line of the myelin lamellae at the site of the radial component somewhat increases the electron density, as compared with that in the other part of the myelin sheath (Figs. 1 to 7 ).

The rows of thicknings in the radial components show two. different patterns of distribution in the myelin sheath; they are sometimes found lying scattered in a relatively large part of the myelin sheath at nearly same intervals of 500 to $600 \AA$, and this type of rows is termed "diffuse type" (Fig. 1), while usually they have a tendency to occur in groups of 2 to 30 or more in number in a small limited part of the sheath, and this is termed "condensed type" (Figs. 2 to 7 ). In the condensed type a regular spacing of about 150 to $270 \AA$ is present between the adjacent rows in each group of the radial components. It has been reckoned that the width of the interperiod line of the myelin lamellae in the site of the thickenings increases from its normal thickness of $20 \AA$ to about. $50 \AA$ and the extent of the thickening along the interperiod line is about 60 to $90 \AA$. The electron density of the thickenings is fairly large as compared with that of the interperiod line which is usually obscure in the non- or weakly-stained sections fixed with $\mathrm{OsO}_{4}$. It should be worthy to note here that the periodic spacing of the myelin lamellar layers increases at the site of the radial components from its normal thickness of about $110 \AA$ to about $130 \AA$. Some of the radial components extend throughout all of the thickness of the myelin sheath, while the others extend for only a part of the myelin lamellar layers; in the latter case, the radial components are usually found in the superficial (external) part of the myelin lamellar layers and lack in the profound (internal) part of the myelin sheath (Figs. $2,6,7)$.

When the radial components are sectioned obliquely to the myelin lamellar membranes, they appear as dense lines which show a parallel array arranged across the thickness of the myelin sheath (Fig. 8). These lines show a width of about 60 to $90 \AA$. Their electron density is larger than that of the period lines of the myelin lamellae.

Strictly speaking, we have found no firm site for the radial components in the myelin sheath, in relation to the other formed elements of the nerve fiber. The most ordinary site for them is in that part of myelin sheath which is lying under the outer loop (tongue process) which is the extension of the processes of the 
oligodendroglial cell (Figs. 1, 5, 6, 7, 8). However, the radial components lie not only under the outer loop, but also are very commonly found in the other parts of the myelin sheath, i. e. in those parts where the myelin sheath is lying near the inner loop (Figs. $1,4)$, coming into contact with another myelin sheath belonging to the adjacent nerve fiber (Fig. 2), or being adjacent to other neuropiles. It has been sornetimes found that the rows of the radial components extend continuously over two adjacent myelin sheaths which come into intimate contact side by side with each other (Fig. 2).

Frequently two or more radial components are found in a thin section of one and the same myelin sheath where the individual groups of the rows of thickenings often run radially through the myelin sheath situated in different directions respectively. There is found no relation between the frequency in appearance of the radial components and the thickness of the myelin sheath or the size of the nerve fibers.

\section{Discussion}

$\mathrm{H}$ o $\mathrm{n}$ jin (1959) in the myelin sheaths from the spinal cords of frogs (Rana nigromaculata nigromaculata), P e te $\mathrm{r}(1961,1962)$ in those from the optic nerves of rats, mice and toads (Xenopus laevis) and $\mathrm{H}$ on jin, Kosaka, Takano and Hiramats u (1963) in those from the optic nerves and the spinal cords of mice (the KH-1 Strain of Mus wagneri var. albula) and frogs (Rana nigromaculata nigromaculata), have demonstrated the radial components respectively. They have already reported that the radial components are revealed as a series of thickenings of the interperiod line in transverse sections of the myelin lamellar layers. Hon ji n, Kosaka, Ta$\mathrm{k}$ a $\mathrm{no}$ and $\mathrm{Hiramatsu}$ (1963) have indicated that the radial components profess to be dense lines passing through the thickness of the myelin sheath when they are cut obliquely to the myelin lamellar membranes, and that this lineal pattern of the radial components in oblique sections results from the electron optical mechanism of thin sections of the myelin sheath which contains many thin layers differing from each other in electron density. The present observations obtained from the optic nerves of frogs (Rana pipiens) confirm the results of these previous researchers, as mentioned above. However, as regards to the distribution of the rows of radial component in the myelin sheath, the present study newly 
points out the existence of the diffuse type of the radial components, besides the condensed type which has been already reported by the previous authors. The diffuse type is scattered in a relatively large part of the myelin sheath and shows a fairly large interval of 500 to $600 \AA$ between the individual radial rows, while the condensed type occurs in a small limited area of the myelin sheath in group of many crowded rows showing a relatively small interval of 150 to $270 \AA$ between each rows and is more frequently found than the diffuse one. The present observations indicate that the layers of the myelin sheath at the local site of the radial component show a slight increase of lamellar periodicity. This supports the findings in other animals by $\mathrm{H}$ on jin, $\mathrm{Kos}$ oka, Takano and $\mathrm{Hira-}$ $\mathrm{m}$ a t s u (1963).

The constant situation of the thickenings in the interperiod lines, the steadily radial arrangement at regular intervals, the confined site of the group of rows in some of the myelin sheaths, the scattered appearance through the entire block of the optic nerve, and the diverse directions of several groups of rows in a same thin section, indicate that the radial component is not a chemical or mechanical artifact caused during the procedures of electron microscopic speciments, but is an image of the true composition and structure of the myelin sheath in the optic nerve of this species of animal. The radial components are frequently found in that part of the myelin sheath which is lying under the cytoplasm of the outer or inner loops which are the processes of the oligodendroglial cell. This indicates that a rôle in myelin metabolism may be estimated for the radial component, but there is no definite finding suggesting this.

\section{Summary}

The radial components of the myelin sheath in the optic nerve of frogs (Rana pipiens) have been studied by electron mincroscopy. The nerves were fixed in $\mathrm{OsO}_{4}$ or $\mathrm{KMnO}_{4}$, embedded in methacrylate or epoxy resin, and, after sectionings, non-stained or stained with either potassium permanganate or plumbite ion method. The results obtained are summarized as follows:

1. The radial components are present in some of the myelin sheath of the optic nerve of the frog, Rana pipiens.

2. The radial components in transverse sections of the myelin lamellar layers appear as a series of small thickenings which are 
present in the interperiod lines of the myelin lamellae and stand in several rows arranged radially across the thickness of the myelin sheath. The dense period line of the myelin sheath at the site of the radial component somewhat increases the electron density. In oblique sections of the myelin lamellae the radial components appear as dense lines which show a parallel array running across the thickness of the sheath.

3. The thickness of the myelin lamellae increases at the site of the radial components.

4. The rows of the thickenings in the radial components show two different patterns of distribution in the myelin sheath; the diffuse and condensed types. The former is scattered in a relatively large part of the myelin sheath and shows a fairly large interval of 500 to $600 \AA$ between the individual rows, while the latter occurs in a small limited area of the sheath in group of many parallel rows showing a relatively small interval of 150 to $270 \AA$ between each rows.

5. Most of the rows of thickenings extend throughout the thickness of the myelin sheath, while some of them extend for only a part of the superficial layers of the myelin lamellae.

6 . Sometimes the rows of the radial components extend continuously over two adjacent myelin sheaths which come into intimate contact side by side with each other.

\section{References}

Gl a uert, A.M. and Gla uert, R.H. 1958: Araldite as an embedding medium for electron microscopy. J. Biophysic. and Biochem. Cytol., 4: 191-194.

Hon jin, R. 1959: Electron microscopic studies on the myelinated nerve fibers in the central nervous system. Acta Anat. Nipponica, $34: 43-44$. (in Japanese)

- K o s a ka, T., Tak a $\mathrm{n}$ o, I. and $\mathrm{H}$ ir a mat s u, K. 1963: Electron microscopy of nerve fibers VII. On the electron dense radial component in the laminated myelin sheath. Okajimas Fol. anat. jap., 39: 39-53.

Karnovsky, M. J. 1961: Simple methods for "staining with lead" at high pH in electron microscopy. J. Biophysic. and Biochem. Cytol., 11: 729-732,

L a w n, A.M. 1960: The use of potassium permanganate as an electron-dense stain for sections of tissue embedded in epoxy resin. J. Biophysic. and Biochem. Cytol., $7:$ 197-198.

L u f t, J.H. 1956: Permanganate-A new fixative for electron microscopy. J. Biophysic. and Biochem. Cytol., 2 : 799-801.

Pa la de, G.E. 1952: A study of fixation for electron microscopy. J. Exp. Med., 95 : 285-298.

Peters, A. 1961: A radial component of central myelin sheaths. J. Biphysic. and Biochem. Cytol., 11 : 733-735. 
1962: Plasma membrane contacts in the central nervous system. J. Anat., London, $96: 237-248$.

Robertson, J.D. 1960 : The molecular structure and contact relationship of cell membranes. Prog. in Biophysics and Biophysic. Chem., 10 : 343-418.

\section{Explanation of Figures}

All illustrations are electron micrographs of thin sections of the optic nerve of frogs, Rana pipiens. The nerve was fixed with $\mathrm{OsO}_{4}$, embedded in epoxy resin and, after sectioning, stained with plumbite ion method.

\section{Plate I}

Fig. 1. Transverse section of myelin sheaths. Note the radial components in form of diffuse type. $\times 120,000$.

Fig. 2. Two nerve fibers sectioned obliquely to their axis. The radial components extend over two adjacent myelin sheaths which come into contact with each other. $\times 100,000$.

\section{Plate II}

Fig. 3. Transverse section of a myelin sheath. Nine rows of thickenings of the radial component are seen. $\times 90,000$.

Fig. 4. Transverse section of two myelin sheaths. The upper myelin sheath holds two groups of radial components. In the right group, the period lines of the myelin lamellar layer becomes much more dense for somewhat long distance in the site of the rows of thickenings. Note the inner loop of the upper nerve fiber. $\times 100,000$.

Fig. 5. A myelin sheath sectioned obliquely to the axis of the nerve fiber. Note the radial components which are running through the thickness of the myelin sheath. $\times 100,000$.

Fig. 6. Radial components of a myelin sheath which is sectioned slightly obliquely to the axis of the nerve fiber. Some of the rows of the radial components extend for only a part of the superficial layers of the myelin lamellae. $\times 100,000$.

Fig. 7. Radial components of a myelin sheath which is sectioned obliquely to the axis of the nerve fiber. The radial components across the thickness at an oblique direction to the lamellae. $\times 100,000$.

Fig. 8. Radial components of a myelin sheath which is sectioned obliquely to the lamellar layers. The radial components appear as dense lines. $\times 100,000$. 
Plate I
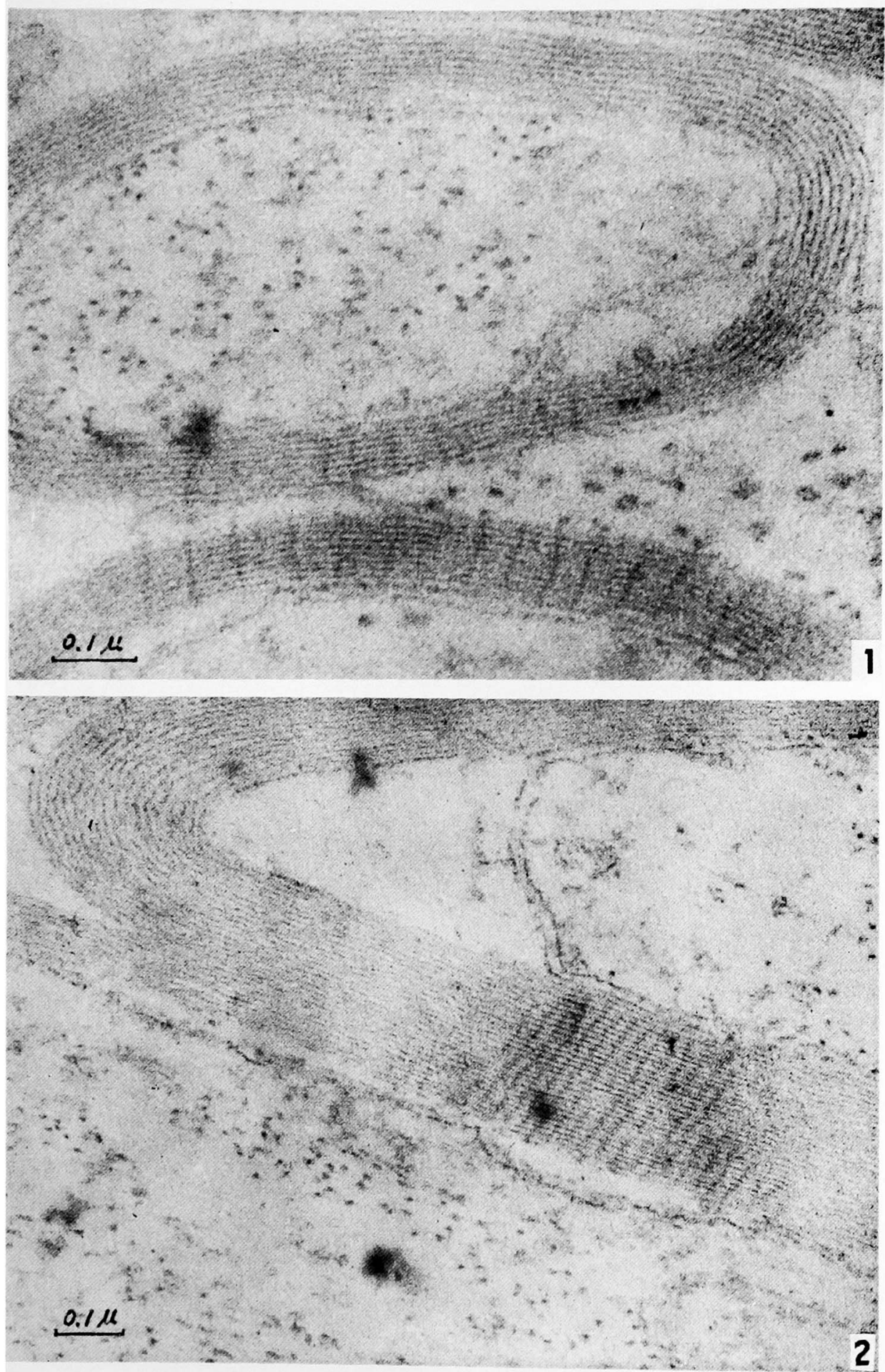

R. Honjin and G. W. Changus 
Plate II
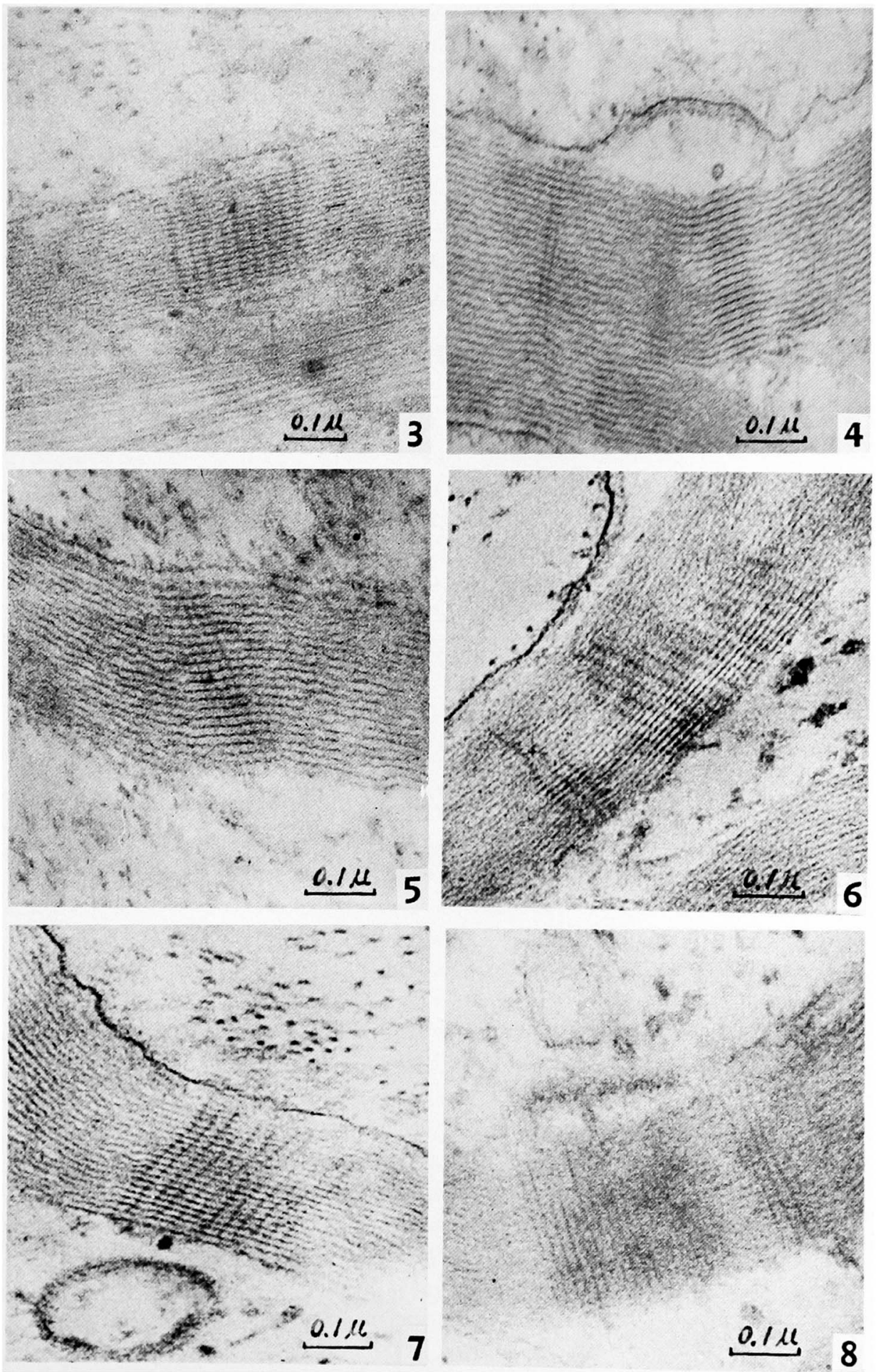

R. Honjin and G. W. Changus 